\title{
Cost analysis of smear microscopy and the Xpert assay for tuberculosis diagnosis: average turnaround time
}

\author{
Lida Jouca de Assis Figueredo[1], Silvana Spíndola de Miranda ${ }^{[2]}$, \\ Lucas Benício dos Santos ${ }^{[1]}$, Caroline Gontijo Gonçalves Manso ${ }^{[1]}$, Valéria Martins Soares ${ }^{[3]}$, \\ Suely Alves ${ }^{[4]}$, Maria Cláudia Vater ${ }^{[4]}$, Afrânio Lineu Kritski ${ }^{[4]}$, Wânia da Silva Carvalho ${ }^{[5]}$, \\ Cristiane Menezes de Pádua ${ }^{[5]}$ and Isabela Neves de Almeida ${ }^{[1]}$
}

\begin{abstract}
[1]. Universidade Federal de Minas Gerais, Laboratório de Pesquisa em Micobactérias, Belo Horizonte, MG, Brasil.
[2]. Universidade Federal de Minas Gerais, Grupo de Pesquisa em Micobacterioses, Faculdade de Medicina, Belo Horizonte, MG, Brasil.

[3]. Fundação Hospitalar do Estado de Minas Gerais, Hospital Júlia Kubistchek, Laboratório de Microbiologia, Belo Horizonte, MG, Brasil.
\end{abstract}

[4]. Universidade Federal do Rio de Janeiro, Programa Acadêmico de Tuberculose, Rio de Janeiro, RJ, Brasil.

[5]. Universidade Federal de Minas Gerais, Faculdade de Farmácia, Belo Horizonte, MG, Brasil.

\begin{abstract}
Introduction: Rapid and accurate tuberculosis detection is critical for improving patient diagnosis and decreasing tuberculosis transmission. Molecular assays can significantly increase laboratory costs; therefore, the average time and economic impact should be evaluated before implementing a new technology. The aim of this study was to evaluate the cost and average turnaround time of smear microscopy and Xpert assay at a university hospital. Methods: The turnaround time and cost of the laboratory diagnosis of tuberculosis were calculated based on the mean cost and activity based costing (ABC). Results: The average turnaround time for smear microscopy was 16.6 hours while that for Xpert was 24.1 hours. The Xpert had a mean cost of USD 17.37 with an ABC of USD 10.86, while smear microscopy had a mean cost of USD 13.31 with an ABC of USD 6.01. The sensitivity of smear microscopy was $42.9 \%$ and its specificity was $99.1 \%$, while the Xpert assay had a sensitivity of $100 \%$ and a specificity of $96.7 \%$. Conclusions: The Xpert assay has high accuracy; however, the turnaround time and cost of smear microscopy were lower than those of Xpert.
\end{abstract}

Keywords: Mycobacterium tuberculosis. Molecular diagnostic. Pulmonary tuberculosis. Cost analysis. Health system.

\section{INTRODUCTION}

Tuberculosis (TB) has existed for millennia and remains a major global health problem ${ }^{1}$. The World Health Organization (WHO) estimated that in 2018, approximately 10 million people had developed TB, and that TB was responsible for an estimated 1.2 million deaths among human immunodeficiency virus (HIV) negative patients and an additional 251 thousand deaths among patients living with HIV $^{1}$.

Brazil is one of 30 countries with a high TB burden, accounting for $84 \%$ of the global total number of cases ${ }^{1}$. In $2018,78,652$ new cases were registered, and only $34 \%$ of them were tested using the

\footnotetext{
Corresponding author: Dra. Silvana Spíndola de Miranda.

e-mail: silvanaspindola@gmail.com

(D) https://orcid.org/0000-0001-7245-4472

Received 17 June 2020

Accepted 24 July 2020
}

Xpert ${ }^{\circledR}$ MTB/RIF assay (Cepheid, Sunnyvale, CA, USA) (Xpert). Moreover, $79 \%$ of the new patients knew their HIV status, and $87 \%$ of the identified cases were pulmonary $\mathrm{TB}^{1}$.

TB typically affects the lungs, but can also spread to other sites ${ }^{1}$. The diagnosis of extrapulmonary TB remains a challenge, since the total number of bacteria in extrapulmonary specimens is often lower than that present in pulmonary specimens ${ }^{2}$. Furthermore, the collection of extrapulmonary material requires invasive procedures, and it is usually difficult to obtain additional samples ${ }^{2}$.

Accurate, rapid detection of TB is critical for improving patient care and decreasing TB transmission ${ }^{1}$. The Xpert assay is an automated molecular test that can detect both TB and rifampicin resistance, generally within two hours after starting the test, with minimal hands-on technical time ${ }^{3}$.

The WHO issued initial recommendations concerning the Xpert test in early $2011^{4}$. In a systematic review, its sensitivity in detecting 
TB in pulmonary samples ranged from $58 \%$ to $100 \%$, whereas the specificity ranged from $86 \%$ to $100 \%{ }^{3}$.

The authors highlighted that the Xpert test is expensive, and that further research is needed to evaluate its use in TB programs and to assess if this investment could help start treatment promptly and improve health outcomes ${ }^{3}$. Despite the WHO recommendations for the use of the Xpert system, sputum smear microscopy remains widely used in many countries for the rapid diagnosis of TB under routine conditions. This method has a variable sensitivity ranging from $32 \%$ to $89 \%$ and a specificity between $85 \%$ and $100 \%$.

TB control programs must balance costs with performance characteristics and the need for rapid results ${ }^{1,3,6}$. Intensive implementation of molecular assays may lead to significant increases in laboratory cost. Selective implementation of molecular assays could be considered for some settings ${ }^{6}$, and accurate and rapid detection of TB is critical for improving patient outcomes (increased cure and decreased mortality rates, additional drug resistance, treatment failure, and relapse) and decreasing TB transmission ${ }^{3}$.

Thus, the aim of this study was to evaluate and compare the costs and average time to completion of smear microscopy and Xpert assay at a teaching hospital.

\section{METHODS}

\section{Study design, variables and study site}

The present study assessed respiratory and non-respiratory specimens $(n=1009)$ that had been received at the Research Laboratory in Mycobacteria of the School of Medicine of the Federal University of Minas Gerais (UFMG), between November 2014 and November 2015. Of these, 141 patients accepted to participate, signed the term of free informed consent, and were included in the study of the accuracy and time of laboratory tests and in the analysis of clinical and sociodemographic characteristics.

Clinical and sociodemographic data were obtained using a standardized questionnaire and a review of the patients' records on their behavior (alcohol and tobacco use), HIV infection, and any abnormalities on pulmonary imaging (chest X-ray or computed tomography). A pattern suggestive of TB was the presence of cavitation, infiltrate in upper lobe and apical segment of the lower lobe or mediastinal enlargement, or increased hilar lymph node or miliary pattern or pleural effusion or confluent parenchymal opacities, confluent parenchymal opacities, characterized as a budding tree ${ }^{7}$.

These patients received medical care at the teaching hospital of the UFMG, a public and general university hospital that conducts educational, research, and medical care activities. This institution consists of one hospital unit and seven outpatient care centers ${ }^{8,9}$. Direct sputum smear examinations that were urgently requested were excluded from clinical specimens, since at the hospital, the Xpert system is not used immediately.

\section{Processing of clinical samples}

The specimens were processed following the standard $\mathrm{N}$-acetyl1-cysteine and sodium hydroxide (NALC/NaOH) method with a final $\mathrm{NaOH}$ concentration of $1 \%{ }^{10}$. After this step, the sediment was resuspended in 1.0 to $1.5 \mathrm{~mL}$ of sterile water and used for smear microscopy, Xpert testing, and culture tests.

Smear microscopy: All smears prepared from decontaminated samples were stained with auramine $\mathrm{O}$ and analyzed by fluorescence microscopy ${ }^{10}$.

$X p e r t^{\circledR} M T B / R I F$ : This assay was performed according to the manufacturer's instructions (Cepheid, Sunnyvale, CA, USA). Briefly, a sample reagent was added in a $3: 1$ ratio to $\geq 0.5 \mathrm{~mL}$ of decontaminated specimen. The closed tube was agitated manually twice during a 15-min incubation at room temperature. Then, $2 \mathrm{~mL}$ of the inactivated sample reagent-sample mixture was transferred to the Xpert test cartridge. The cartridges were inserted into the Xpert device, and the automatically generated results were read after $90 \mathrm{~min}^{3,11}$.

\section{Average time: smear microscopy and Xpert}

To calculate the average time to the release of test results, we took into consideration the hour when the samples were received in the collection sector and the hour when test results were released in the system. This period includes the times of receipt of samples, internal registration, the process of decontamination/centrifugation of the samples, separation of the aliquots for each diagnostic test, execution of the tests, release of results by the technician, and release of the results into the computerized system of the hospital.

The times during which tests were performed urgently via direct smear, at night, on weekends and holidays were not considered; only those performed routinely with NALC/NaOH were considered.

\section{Cost analysis}

The cost analysis of the TB laboratory diagnosis was based on the mean cost and activity based costing (ABC). The mean cost was calculated by dividing the total costs by the quantity produced over a determined period of time ${ }^{12}$, which considered the total number of examinations performed in a month. The ABC principle is suitable for complex organizations, such as hospitals, where products use consumer resources in a highly heterogeneous manner ${ }^{9,12}$, therefore covering as many direct and indirect costs as possible through cost drivers. The component cost evaluation and calculations were performed as described by Almeida et al. 2017 and the costs were expressed in USD, using the conversion rate of USD $1.00=\mathrm{R} \$ 4.03$, as established by the Central Bank of Brazil in 2019 (https://www.bcb.gov.br/) $)^{9,13,14}$.

\section{Statistical analysis}

Descriptive analysis was performed to characterize the study population concerning the selected variables. Measures of central tendency and dispersion were used for continuous variables, and absolute and relative frequencies for categorical variables.

The sensitivity and specificity of $X p e r t^{\circledR} M T B / R I F$ and smear microscopy with their $95 \%$ confidence intervals (CIs) were estimated, comparing the results to those of the phenotypic identification test as a standard method ${ }^{10}$. All analyses were performed using SAS version 9.4 (SAS Institute Inc., Cary, NC, USA). 


\section{Ethical considerations}

The study protocol was approved by the Research Ethics Committee of the UFMG (protocol numbers CAAE11821913.6.000.5257 and CAAE 0223.2412.7.1001.5149, and DEPE/HC protocol number 139/12).

\section{RESULTS}

The study included 141 patients' clinical samples: 100 pulmonary specimens and 41 extrapulmonary specimens (Figure 1); 66\% (93/141) of the patients were inpatients and 34\% (48/141) were outpatients.

The sociodemographic characteristics of these patients are presented in Table 1. Most patients were male, aged 46 to 60 years (median=46.0), single, had non-white skin, had nine or fewer years of formal education, and 25 had pulmonary TB. Between $20 \%$ and $40 \%$ of the patients were reported to be current users of alcohol and tobacco, respectively. Regarding radiological patterns, 27\% presented presumed TB abnormalities.

Accuracy assessment of the smear microscopy revealed a sensitivity of $42.9 \%$ (95\% CI, 24.5-61.2) and a specificity of $99.1 \%$

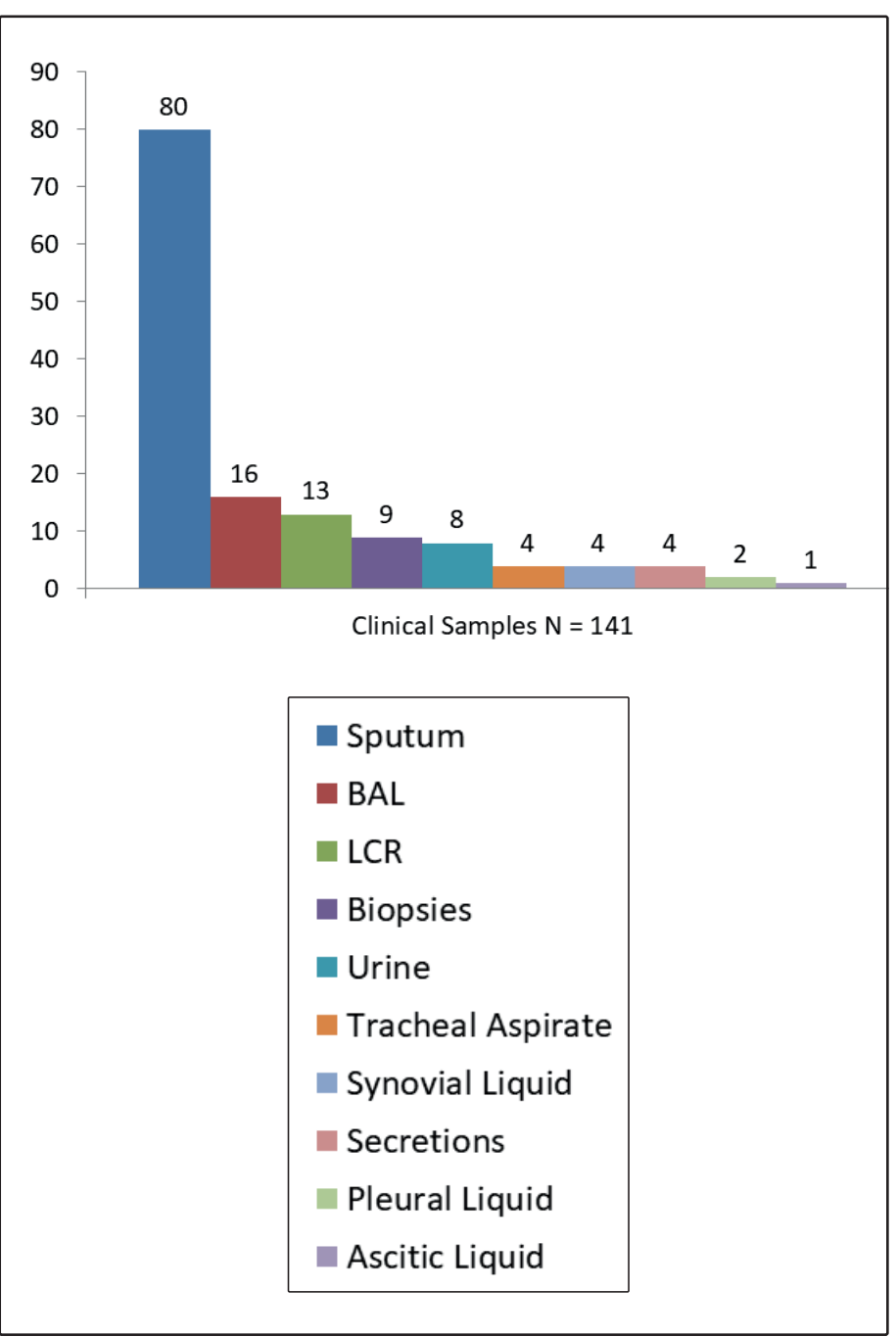

FIGURE 1: The distribution of clinical samples.
TABLE 1: Distribution of sociodemographic characteristics $(n=141)^{*}$

\section{Frequency}

Variables

Total number of patients

n

Sociodemographic

Age, years

18-35

39

28.7

36-45

19.9

46-60

27.2

61-88

Gender

Male

83

58.9

Skin color

Non-white

80.0

White

20.0

Education, years

Illiterate

8.2

$\leq 9$

63.9

$\geq 10$

27.9

Marital status

Married

48.3

Single

Behavioral

Alcohol use (CAGE)

Positive

40.7

Negative

59.3

Tobacco use

Never

Current

Ever

Clinical

Prescribed TB therapy

RHZE

Special regimen

None

HIV status

Positive

Negative

26.2

Unknown result/not tested

55.3

Radiological patterns

Suggestive of TB

Without abnormalities

27.0

Other abnormalities

5.0

26.2

Not performed

41.8 
(95\% CI, 97.2-100.0). The accuracy assessment of the Xpert assay revealed a sensitivity of $100 \%(95 \% \mathrm{CI}, 1.00-1.00)$ and a specificity of $96.7 \%$ (95\% CI, 93.53-99.88).

Positive Xpert assays and negative culture test results were observed for the samples of two patients in retreatment, while 11 positive culture test results for nontuberculous mycobacteria (NTM) were not detected by the Xpert system.

The average time to smear microscopy results was 16.6 hours and the median time was 9.3 hours (standard deviation, 15.1 hours; range, 1.2-48 hours). The mean time to completion of the Xpert assay was 24.1 hours, with a median time of 24 hours (standard deviation, 18.2 hours; range, $2-72$ hours).

\section{Cost analysis}

The mean cost of the Xpert assay was USD 17.37 with an ABC value of USD 10.86, while the mean cost of the smear microscopy was USD 13.31 with an ABC value of USD 6.01. Proportionally, the impact of cost components of smear microscopy was lower than that of Xpert, except for the human resources component. The component cost that impacted the mean costs and $\mathrm{ABC}$ values of Xpert and smear microscopy are described in Figure 2.

\section{DISCUSSION}

The study revealed that the average time to completion of the Xpert assay result was longer than that of sputum smear microscopy. This is because the slide is read immediately, but the sample undergoes processing in the Xpert system. In addition, the fact that in our laboratory we use the equipment with only four modules might also play a role. Nevertheless, although smear microscopy is cheaper than the Xpert assay, the latter is more accurate.

The advantage of the Xpert system is that it offers the possibility for a fully automated test after insertion of the clinical specimen into the cartridge. Assay steps (DNA extraction, amplification, and detection) are independent of manual technical interventions, thus minimizing analytical errors and improving quality control ${ }^{3}$.

The sensitivity and specificity values found in this study $(100 \%$ and $96.7 \%$, respectively) are similar to the results of a meta-analysis that included 27 studies with a total of 9500 specimens analyzed using the Xpert system, and which presented a range of sensitivity between $58 \%$ and $100 \%$ and specificity between $86 \%$ and $100 \%{ }^{3}$.

The specificity was found to be lower than the sensitivity due to the presence of $M$. tuberculosis complex DNA in two cases of treatment control patients who presented negative culture tests. Therefore, the recommendation not to use the Xpert system for treatment control should be reinforced, except for probable TB in new cases, if resistance to rifampicin or multidrug resistance is suspected.

In this study, the Xpert system did not show false positive results among NTM isolates, mainly due to the increase in prevalence of disease-associated NTM, as has already been described by other authors ${ }^{15-17}$. Therefore, NTM should be suspected in conditions where smear microscopy tests yield positive results and are undetected by the Xpert assay ${ }^{18}$. Our data agree with the results of a meta-analysis that evaluated 14 studies including 180 NTM cases and showed that the false positivity of the Xpert assay for these pathogens was only $0.6 \%{ }^{3}$.

The average times extrapolated from the results (16.3 and 24 hours for smear microscopy and Xpert assay, respectively) are due to the fact that the laboratory does not work at night, on weekends, and holidays with processing of samples with $\mathrm{NALC} / \mathrm{NaOH}$; thus, the clinical samples are stored for the test to be carried out on the next business day. The recommendation of the Ministry of Health and reinforced by other authors is that routine results should be released within 24 hours ${ }^{11,18,20,21}$, which was seen from the mean of our results.

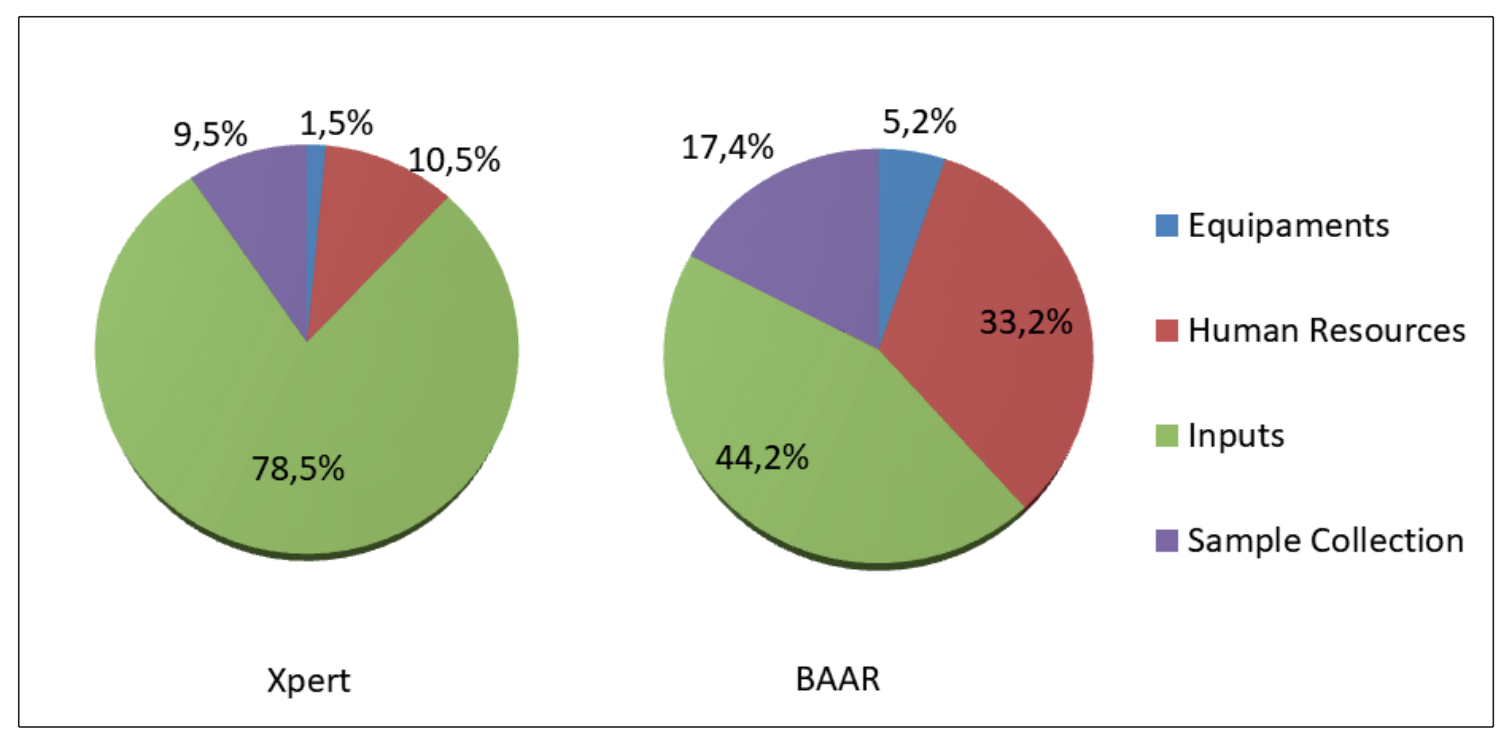

FIGURE 2: The impact of $A B C$ components. 
The $\mathrm{ABC}$ value of the Xpert assay was higher than that of smear microscopy and the input was the component with the greatest impact. However, it should be noted that the Xpert system is subsidized in Brazil ${ }^{22}$. Efforts are being made to increase the coverage area of the Xpert system worldwide through subsidies to make it available in developing and underdeveloped countries, where health systems work under heavy economic constraints ${ }^{22,23}$.

This is mainly due to the demand of exclusive inputs from the supplier that are imported and undergo exchange variation, which is a dynamic and sensitive variable to the economic scenario of each country ${ }^{24}$.

When incorporating new technology and comparing it with already existing technology, it is important to evaluate all operational and technical aspects. Managers need to be aware of the relevant outcomes of TB treatment and bacillus transmission in the community when evaluating the advantages and disadvantages of incorporating new diagnostic tests ${ }^{25,26}$.

The results described in this paper should alert Brazilian researchers to the need for the development and validation of a national molecular test. Although the Xpert system has high accuracy in the diagnosis of TB, national technological independence is necessary to ensure that cost is not a variable that hinders or even impedes the incorporation of a tool that is effective for the rapid and accurate diagnosis of $\mathrm{TB}^{22,27}$.

The results should not be extrapolated to other scenarios owing to differences in the components analyzed. Thus, each site must perform its own analysis.

In conclusion, the Xpert assay has high accuracy; however, the time to sputum smear test results and the $\mathrm{ABC}$ value were lower than those observed for the Xpert system.

\section{ACKNOWLEDGMENTS}

We are grateful to the UFMG Postgraduate Program in Infectology and Tropical Medicine, to the Pro Rectory Research/ UFMG, and to the Brazilian Tuberculosis Network (REDE TB). We are also grateful to the Minas Gerais Research Support Foundation (FAPEMIG) and the National Research Council.

\section{FINANCIAL SUPPORT}

This research was funded by the Minas Gerais Research Support Foundation (FAPEMIG) process numbers: APQ-03266-13 and APQ-00094-12 and the National Research Council (CNPq) (process numbers: 446796/2014 and 310174/2014-7) and the Coordination of Improvement of Higher Level Personnel of the Ministry of Education of Brazil (CAPES). Dr Isabela Almeida received funds for this work under a pos-doctoral scholarship (process - CNPq / INCT 465318/2014-2).

\section{AUTHORS' CONTRIBUTIONS}

LJAF: data collection, development of experiments, building the database, monitoring cost analysis, $\mathrm{PhD}$ student, and article writing. SSM: study conception and design, promotion of study financing, data analysis, and article writing. LBS: data collection, typing and conferencing of the database, and article writing. VMS: data collection, typing and conferencing of the database, and article review. SA: cost calculation, cost chain modeling, database conferencing, and article review. MCV: co-coordination of cost study, cost chain modeling, database conferencing, and article writing. ALK: study conception and design, and article review. WSC: study conception and design, promotion of study financing, data analysis, and article writing. CMP: statistical analysis, adjustment of the database, and article writing. INA: coordination of the data collected, adjustment of the database, modeling of the cost analyses, data analysis, and article writing. All the authors read and approved the final manuscript.

\section{CONFLICTS OF INTEREST}

The authors declare no conflicts of interests.

\section{REFERENCES}

1. World Health Organization (WHO). Global tuberculosis report 2019. Geneva: WHO; 2019. 194p.

2. Hillemann D, Rüsch-Gerdes S, Boehme C, Richter E. Rapid Molecular Detection of Extrapulmonary Tuberculosis by the Automated GeneXpert MTB/RIF System. J Clin Microbiol. 2011;49(4):1202-5.

3. Steingart KR, Schiller I, Horne DJ, Pai M, Boehme CC, Dendukuri N. Xpert ${ }^{\circledR}$ MTB/RIF assay for pulmonary tuberculosis and rifampicin resistance in adults. Cochrane Database Syst Rev. 2014; 21:CD009593.

4. Molicotti P, Bua A, Zanetti S. Cost-effectiveness in the diagnosis of tuberculosis: choices in developing countries. J Infect Dev Ctries. 2014;8(1):24-38.

5. Sagili KD, Muniyandi M, Nilgiriwala KS, Shringarpure KS, Satyanarayana S, Kirubakaran R, et al. Cost-effectiveness of GeneXpert and LED-FM for diagnosis of pulmonary tuberculosis: A systematic review. PLoS One. 2018;13:e0205233.

6. Shah M, Chihota V, Coetzee G, Churchyard G, Dorman S. Comparison of laboratory costs of rapid molecular tests and conventional diagnostics for detection of tuberculosis and drug-resistant tuberculosis in South Africa. BMC Infect Dis. 2013;13:352-58.

7. Capone D, Jansen JM, Lopes AJ, do Couto Sant'Anna C, Soares MO, dos Santos Pinto R, et al. Diagnóstico por imagem da tuberculose. Pulmão RJ. 2006;15(3):166-74.

8. Universidade Federal de Minas Gerais (UFMG). Hospital das Clínicas [Internet]. Estrutura Física: Conheça o HC; 2019. [updated 2018 September 19; cited 2019 Sep 29] Available from: http://www2.ebserh. gov.br/web/hc-ufmg/infraestrutura.

9. de Almeida IN, Figueredo LJA, Soares VM, Vater MC, Alves S, Carvalho WS, et al. Evaluation of the Mean Cost and Activity Based Cost in the Diagnosis of Pulmonary Tuberculosis in the Laboratory Routine of a High-Complexity Hospital in Brazil. Front Microbiol. 2017;8:249.

10. Ministério da Saúde (MS). Secretaria de Vigilância em Saúde. Manual nacional de vigilância laboratorial da tuberculose e outras micobactérias. Brasília: MS; 2010. 190 p.

11. World Health Organization (WHO). Automated Real-Time Nucleic Acid Amplification Technology for Rapid and Simultaneous Detection of Tuberculosis and Drug Resistant Tuberculosis: Policy Statement. Geneva: WHO; 2013. 10 p.

12. Ministério da Saúde (MS). Secretaria de Ciência, Tecnologia e Insumos Estratégicos. Departamento de Economia da Saúde. Manual Técnico de Custos - Conceitos e Metodologias. Brasília: MS; 2006. 1-71p. 
13. Scherer LC, Sperhacke RD, Ruffino-Netto A, Rossetti ML, Vater C, Klatser P, et al. Cost-effectiveness analysis of PCR for the rapid diagnosis of pulmonary tuberculosis. BMC Infect Dis. 2009;9:216-20.

14. Banco Central do Brasil (BC). Cotações e Boletins, 2019 [Internet]. [updated 2020 May 19; cited 2020 May 19]. Avaliable from: https:// www.bcb.gov.br/acessoinformacao/legado?url=https:\%2F\%2Fwww4. b cb.gov.br $\% 2$ Fpec $\% 2$ Ftaxas $\% 2$ Fport $\% 2$ Fptax n pesq. asp\%3Fid\%3Dtxcotacao.

15. Fernandes HMZ, Conceição EC, Gomes KM, da Silva MG, Dias RCS, Duarte RS. Recovery of Non-tuberculous Mycobacteria from Water is Influenced by Phenotypic Characteristics and Decontamination Methods. Curr Microbiol. 2020;77(4):621-31.

16. Bryant J, Grogono D, Rodriguez-Rincon D, Everall I, Brown KP, Moreno $\mathrm{P}$, et al. Emergence and spread of a human-transmissible multidrugresistant nontuberculous mycobacterium. Science. 2016;354(6313):75157.

17. Floto R, Olivier K, Saiman L, Daley CL, Herrmann JL, Nick JA, et al. US Cystic Fibrosis Foundation and European Cystic Fibrosis Society consensus recommendations for the management of non-tuberculous mycobacter in individuals with cystic fibrosis. Thorax. 2016; 71:Suppl 1:i1-22.

18. Ministério da Saúde (MS). Secretaria de Vigilância em Saúde. Manual de Recomendações para o Controle da Tuberculose no Brasil. Departamento de Vigilância das Doenças Transmissíveis. Brasília: MS; 2019. 55-63p

19. Rachow A, Zumla A, Heinrich N, Rojas-Ponce G, Mtafya B, Reither K, et al. Rapid and accurate detection of Mycobacterium tuberculosis in sputum samples by Cepheid Xpert MTB/RIF assay-a clinical validation study. PLoS One 2011;6(6):e20458.
20. Chaisson LH, Duong D, Cattamanchi A, Roemer M, Handley MA, Schillinger D, et al. Association of Rapid Molecular Testing With Duration of Respiratory Isolation for Patients With Possible Tuberculosis in a US Hospital. JAMA Intern Med. 2018;178(10):1380-88.

21. Chaisson LH, Roemer M, Cantu D, Haller B, Millman AJ, Cattamanchi A, et al. Impact of GeneXpert MTB/RIF assay on triage of respiratory isolation rooms for inpatients with presumed tuberculosis: a hypothetical trial. Clin Infect Dis. 2014;59(10): 1353-60.

22. Pinto MFT, Steffen R, Entringer A, Costa ACCD, Trajman A. Budget impact of the incorporation of GeneXpert MTB/RIF for diagnosis of pulmonary tuberculosis from the perspective of the Brazilian Unified National Health System, Brazil, 2013-2017. Cad Saude Publica. 2017;33(9):e00214515.

23. Kaur R, Kachroo K, Sharma JK, Vatturi SM, Dang A. Diagnostic Accuracy of Xpert Test in Tuberculosis Detection: A Systematic Review and Meta-analysis. J Glob Infect Dis. 2016;8(1):32-40.

24. Barreira D. Os desafios para a eliminação da tuberculose no Brasil. Epidemiol Serv Saúde. 2018;27(1):e00100009.

25. Acuna-Villaorduna C, Vassall A, Henostroza G, Seas C, Guerra H, Vasquez L, et al. Cost-Effectiveness Analysis of Introduction of Rapid, Alternative Methods to Identify Multidrug-Resistant Tuberculosis in Middle-Income Countries. Clin Infect Dis. 2008;47(4):487-95.

26. Laranjeira de Oliveira F; Petramale CA. A avaliação econômica em saúde na tomada de decisão: a experiência da CONITEC. BIS 2013;14(2):165-70.

27. Drobniewski F, Cooke M, Jordan J, Casali N, Mugwagwa T, Broda A, et al. Systematic review, meta-analysis and economic modelling of molecular diagnostic tests for antibiotic resistance in tuberculosis. Health Technol Assess. 2015;19(34):1-188. 